\title{
A Telerehabilitation System based on Wireless Motion Capture Sensors
}

\author{
Pedro Macedo ${ }^{1,2}$, José A. Afonso ${ }^{1}$, Luis A. Rocha ${ }^{1}$ and Ricardo Simoes ${ }^{2,3,4}$ \\ ${ }^{l}$ Department of Industrial Electronics, University of Minho, Guimarães, Portugal \\ ${ }^{2}$ Institute for Polymers and Composites IPC/I3N, University of Minho, Guimarães, Portugal \\ ${ }^{3}$ Life and Health Sciences Research Institute (ICVS/3Bs), University of Minho, Braga, Portugal \\ ${ }^{4}$ School of Technology, Polytechnic Institute of Cávado and Ave, Barcelos, Portugal \\ \{pmacedo,jose.afonso,lrocha\}@dei.uminho.pt,rsimoes@dep.uminho.pt,rsimoes@ipca.pt
}

\begin{abstract}
Keywords: Wireless Sensor Networks, Motion Capture, Wearable Sensors, Home-based Rehabilitation.
Abstract: $\quad$ The constant growth of the elderly population in the world creates new challenges and opportunities in health care systems. New technological solutions have to be found in order to meet the needs and demands of our aging society. The welfare and quality of life of the elderly population must be a priority. Continuous physical activity will play an important role, due to the increase of the retirement age. However, physiotherapy can be expensive, even when the desire movements are autonomous and simple, also requires people to move to rehabilitation centres. Within this context, this paper describes the development and preliminary tests of a wireless sensor network, based on wearable inertial and magnetic sensors, applied to the capture of human motion. This will enable a personalized home-based rehabilitation system for the elderly or people in remote physical locations.
\end{abstract}

\section{INTRODUCTION}

Developed societies nowadays face an emergent and possibly problematic situation, if not addressed properly: the growth ratio of elderly population compared with the total population. This continuous growth is changing the demographic structure of societies, with lower birth rates and increasing life expectancy (Stula, 2012). This demographic change (Linz and Stula, 2012) imposes new challenges in order to create services and products for ambient assisted living (AAL). These products will enable the creation of better life conditions for the older generation in their environment by increasing their self-confidence, autonomy and mobility (Sun et al., 2009) (Fuchsberger, 2008) (Kleinberger et al., 2007).

The monitoring of human body movements, body kinematics, is a growing research field in areas such as health care, entertainment and sports. Nevertheless when creating a wireless sensor network (WSN) to monitor human body motion, several factors must be taken into account in order to assure a reliable operation. The authors in (Hadjidj et al., 2012) describe the technical challenges faced when creating a WSN for rehabilitation applications and review existing projects. Several challenges need to be addressed on these systems, such as the fixation of the sensor node, energy efficiency (normally the sensors are energy constrained), the usually high amount of generated data per sensor (high sampling rate) and the impact of the human body on the signal propagation (the wireless signal propagation suffers from diffraction around the body and reflection from the body). Human motion tracking systems are usually classified as either visual, by the combination of data from several cameras to achieve a 3D location of the patient's body and limbs (Moeslund and Granum, 2001); (Wang et al., 2003), or non-visual, such as the use of wearable sensors.

This paper focuses on the non-visual method, and describes the development of a wireless sensor network, based on wearable inertial and magnetic sensors. When compared to a camera strategy, this method has the advantages of higher flexibility and mobility, as it can be used in uncontrolled environments, without lighting and line of sight concerns (Aminian and Najafi, 2004).

The capabilities and limitations of this kind of electronic sensors, when applied to the analysis of the human posture and movements, are highlighted 
in (Wong et al., 2007). Several authors have also underlined the high performance of inertial and magnetic sensors, when applied to body kinematics measurement. Accelerometers in (Farella et al., 2008) are used to exploit their capabilities in terms of body posture recognition in dynamic everyday life activities. A protocol to measure the upper limb kinematics using the Xsens MT9B technology was developed in (Cutti et al., 2007), with the purpose of measuring its accuracy (through the protocol) during movements of clinical relevance. The benefits of physical activity are exposed in (Steffen et al., 2011), where the authors propose a personalized, home-based exercise trainer for elderly people. The captured movements are evaluated and compared to a prescribed exercise; the purpose is to assist the user to correctly perform the proposed movements.

The purpose of this paper is to present the current status of a wireless posture monitoring system, based on wearable inertial and magnetic sensors, applied to rehabilitation.

This paper is organized as follows. The next section presents an overview of all components of the implemented system. In the third section, the method of calculation of the orientation angles is presented. Experimental results concerning a rehabilitation session are presented in section 4 . Section 5 tackles future work and presents some preliminary conclusions.

\section{SYSTEM OVERVIEW}

The electronic system of this posture monitoring WSN is composed by a base station connected to a PC and multiple nodes that collect sensory information from the user. Each sensor module is firmly attached to one body segment which we want to monitor. The readings from the inertial and magnetic sensors are continuously collected and sent wirelessly to the base station, which forwards them to the PC through a serial connection. The information processing in the PC allows the calculation, in real-time, of the $3 \mathrm{D}$ orientation of the module, expressed by the pitch, roll and yaw angles. The structure of this system is represented in Figure 1.

Communication between the base station and the sensor nodes is made using the CC2530, from Texas Instruments (Texas Instruments, 2009). The CC2530 is a true system-on-chip ( $\mathrm{SoC}$ ) solution for IEEE 802.15.4 applications (IEEE Std 802.15.4, 2006) which integrates an 8051 based microcontroller and an 802.15.4 transceiver working in the licence-free $2.4 \mathrm{GHz}$ frequency band.

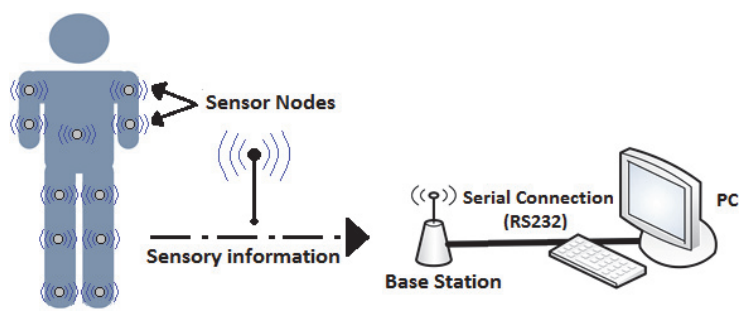

Figure 1: System components.

The Enhanced Low Power Real Time (eLPRT) protocol (Afonso et al., 2011), a MAC protocol that was designed to optimize the quality of service (QoS) provisioning and the bandwidth utilization efficiency, is used to control the communication through the wireless medium.

\subsection{Base Station Architecture}

The base station is responsible for coordinating operation of the wireless sensor network. Its responsibilities include: associate new sensor nodes to the network; allocate time slots on the eLPRT superframe for nodes to transmit; and keep the synchronization in the network, with the help of the periodic transmitted beacons.

The base station is powered by the PC, so its energy consumption is not a concern. The architecture of the base station is shown in Figure 2. The USART (Universal Synchronous Asynchronous Receiver Transmitter) and Radio peripherals are controlled by the CC2530 with the assistance of the DMA (Direct Memory Access) subsystem.

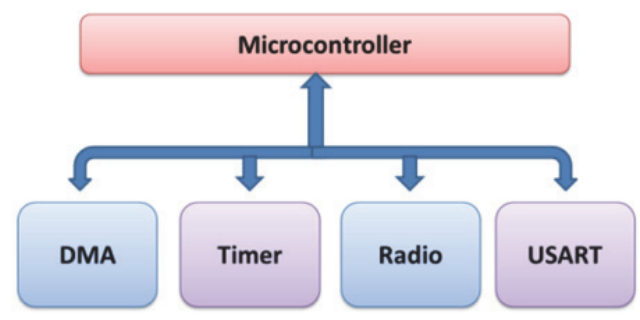

Figure 2: Base station architecture.

Figure 3 presents the hardware of the base station, which is composed by a CC2530EM (Evaluation Module) attached to a SmartRF05EB (Evaluation Board), both from Texas Instruments. 


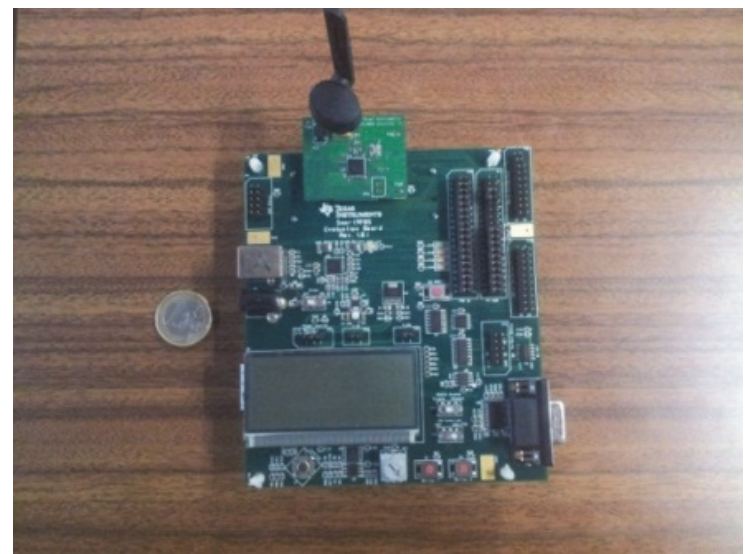

Figure 3: Base station.

\subsection{Sensor Node Architecture}

The data messages between each sensor node and the base station are transmitted periodically according to the superframe period set by the user's application. The number of sensor readings in each period is also set by the user. These multiple readings are aggregated into a single message and sent to the base station. Currently a superframe period of 100 milliseconds and 3 sensor readings is in use. This gives us a rate of 30 frames per second, a typical value for motion capture systems (Claypool et al., 2006).

The sensor board contains 3-axis inertial and magnetic sensors, which measure variations in the gravitational and magnetic field forces, respectively. It is due to these variations that it is possible to calculate the orientation of the sensor node. The sensor node architecture is shown in Figure 4.

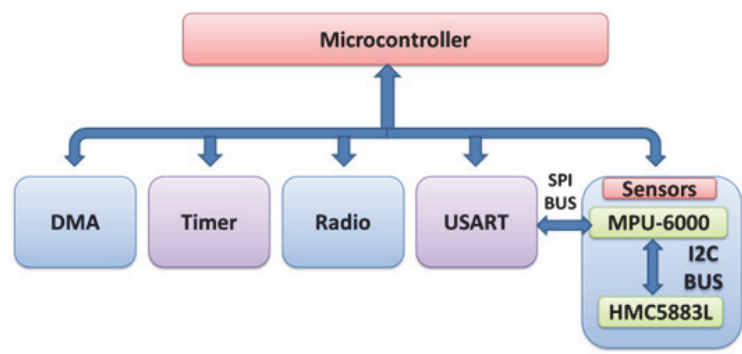

Figure 4: Sensor node architecture.

The sensory information is collected through the SPI bus (serial peripheral interface) and sent to the base station using the eLPRT protocol. Detailed information about the sensors present in the next section.

\subsubsection{Sensor Node Prototype}

The sensor board prototype is illustrated in Figure 5, with the bottom layer on the left side and the top layer on the right side. The sensor board is connected to a CC2530EM module through two 20-pin header connectors.

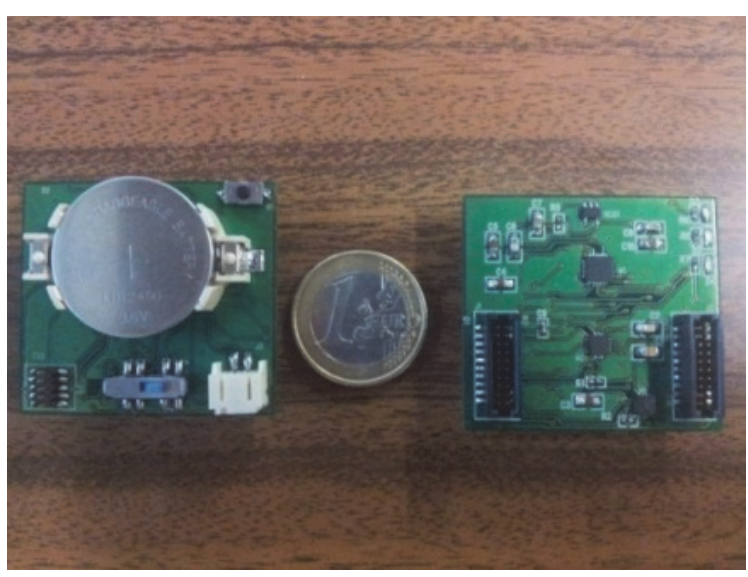

Figure 5: Sensor board.

There are two sensors present in the sensor board. The MPU-6000 Motion Processing Unit from InvenSense, which has an embedded 3-axis MEMS gyroscope (not used at the moment), a 3-axis MEMS accelerometer and a digital motion processor (DMP) hardware accelerator engine with an auxiliary $\mathrm{I}^{2} \mathrm{C}$ port that interfaces to a third party digital sensor, such as a magnetometer. The embedded 16-bit accelerometer has a digital-output triple-axis userprogrammable full-scale range of $\pm 2 \mathrm{~g}, \pm 4 \mathrm{~g}, \pm 8 \mathrm{~g}$ and $16 \mathrm{~g}$, a selectable output data rate from $4 \mathrm{~Hz}$ to $1 \mathrm{kHz}$ and a sensitivity of $16.384 \mathrm{LSB} / \mathrm{g}$ at $\pm 2 \mathrm{~g}$.

The auxiliary $\mathrm{I}^{2} \mathrm{C}$ bus allows the MPU-6000 to directly access the data registers of an external digital sensor. In the case of this prototype, the Honeywell 3-axis Digital Compass IC HMC5883L is used. Designed for low-field magnetic sensing, it contains a 12-bit ADC that enables 1 to 2 degrees compass heading accuracy; with a resolution of 5 milliGauss in \pm 8 gauss fields.

\subsection{PC Software}

In the proposed system, the component with the greatest relevance to the user is the PC application. This application mediates between the user and the base station; it can send commands and receive information sent by the sensor nodes attached to the body segments. After receiving the data from the sensors, it calculates the angles of rotation and then 
presents the movement of the user's body in real-time on a $3 \mathrm{D}$ model of the human body. The software provides means to create rehabilitation session files, where user information and motion angles are stored. These session files can later be employed to evaluate the user's progress. The referred application, Figure 6, was created in the object-oriented programming language Java, due to its portability between operating systems.

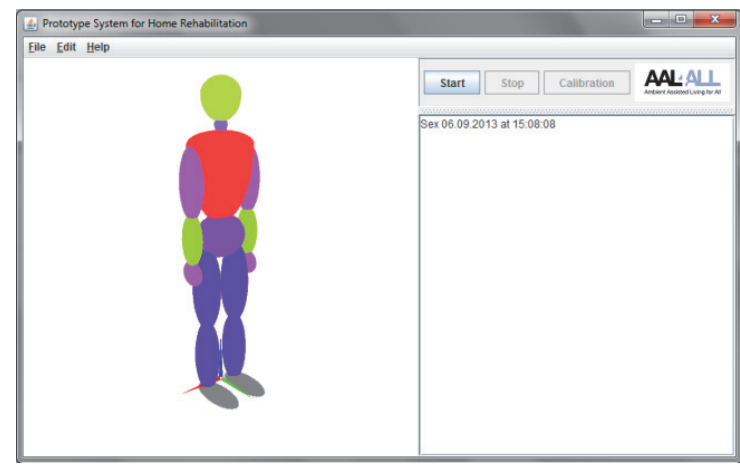

Figure 6: Application interface.

One sensor node per monitored segment is necessary to measure the movement, with three degrees of freedom (DOF), expressed by the Euler angles pitch, roll and yaw. The complete model consists on fifteen rigid body segments: pelvis, torso, head, upper arms, forearms, hands, upper legs, lower legs and foots; connected by virtual joints.

The implementation of this model was made using the Java OpenGL (JOGL), which is a wrapper library that allows OpenGL to be used within the Java environment.

The system was designed to be as intuitive and easy to handle by the user as possible. There are two main components to take into account, in order to have the system in full operation mode: the configuration of the network (configuration of base station and sensor nodes), started automatically by the program when the Start button is pressed; and the calibration of the sensor nodes, that is, the process of finding orientation and placement of the multiple sensor nodes attached to body segments. These components are described in the next section.

\subsubsection{Configuration and Calibration}

Within the application folder, there are two configuration files of utmost importance:

- Configuration File: Herein lay the configuration parameters of sensor registers present in the sensor board. Although there is a default configuration stored in the CC2530 microcontroller, which is then loaded to the sensors, the configuration parameters can be altered, maybe not by the end user, but by a medical specialist (physiotherapist) at the beginning of the treatment.

- Calibration File: This calibration file corresponds to maximum and minimum values of the accelerometer and magnetometer readings. These values are compared to the actual readings of the sensors, for normalization, producing outputs in the range of -1 to 1 , as in ' $\mathrm{g}$-forces (in the case of the accelerometer, this happens when no external force besides gravity is present). One calibration file for each sensor node in the network is required, since not all the sensors give the same readings. This file is generated by a different application. For a better functionality of the system, this calibration should be done at the operation location.

\subsubsection{Program Execution Steps}

In the application's main window, shown in Figure 6, there is a Start button that, when pressed, initiates the configuration of the system. The configuration is divided in the following steps:

- Serial Port Detection: The connection between the PC and the base station is made through a serial port interface, so at this point is fundamental to determine which COM port is associated to the base station. To achieve this, the application starts by discovering all the COM ports available in the $\mathrm{PC}$, and then sends a command through them. The COM port which answers according to what is expected is the port connected to the base station. This process enables the user to use any available port in the $\mathrm{PC}$, which is important with equipment such as a laptop, where USB to serial converters are used.

- Open Configuration File: Opening the configuration file and saving its parameter values (sensors registers) on the application. This file is common to all the sensor nodes in the network. The superframe period and number of samples per period are also configured here. These two parameters combined give us the sampling rate of the sensor readings.

- Configure Superframe Period in the Base Station: A command containing the superframe period is sent to the base station. Immediately after receiving this value, it starts sending beacons at that interval to the network. These beacons allow the synchronization between the 
sensor nodes and the base station.

- Turn on the Sensor Nodes: A notification message appears at the application's main window, alerting that the sensor modules can be turned on by the user. When activated, the sensor node will become associated to the base station. After that, the base station allocates a time slot in the superframe, according to the eLPRT protocol, for the sensor to transmit the data.

- Acquire Associated Nodes Information: A command message is sent to the base station, enquiring for the number of associated sensor nodes and their respective physical address.

- Configure Sensor Nodes: After the discovery of the physical addresses of all the nodes in the network, the application sends the configuration of the sensors registers, superframe period and number of samples to each sensor node (through the base station).

- Data Acquisition: Since the sensor modules are now configured, the application sends a start command message to the base station, for the sensor nodes to begin their readings at the defined sampling rate. The base station broadcasts the message to the network.

The Stop button sends a command to stop data acquisition at the sensor nodes. After that, the link between base station and application is closed.

After these steps are executed, it is necessary to discover the orientation of the sensor nodes (Section 2.3.3) and their body placement (Section 2.3.4). This is achieved by pressing the Calibration button. This action leads to the appearance of the Calibration window. Note that after a calibration is performed, these orientation and body placement values are stored in a file. The purpose of this setting is to facilitate the modules configuration by the user, who can get the stored configuration by pressing the Last Know Configuration button (Calibration window).

\subsubsection{Node Orientation Discovery}

The wireless sensor nodes are attached to the body segments with Velcro straps, always with the antenna opposed to the body, in order to minimize the body effects in the wireless propagation signal, and placed sideways when attached to legs or arms.

Firstly, the user is asked to stand up straight and be still, as depicted in the 3D model, for a period of time. During that amount of time, the API of the application is collecting raw data from the sensor nodes and through them calculating the Euler angles. The estimation of the pitch and roll angles is made using the gravity component obtained from the measurement of the accelerometer, while the yaw is determined from the measures from the electronic compass unit (magnetometer).

The three coordinate rotations in a determined sequence describe the orientation of the module; the rotations of the Euler angles are discussed in Section 3. Once the final rotation matrix is calculated, it is multiplied by the gravitational vector $(0,0,1)$ in $\mathrm{x}, \mathrm{y}$ and $\mathrm{z}$ coordinates, respectively. This multiplication gives the weight of the module orientation on each axis. With this weight and the normalized raw values of the sensors, the orientation of the sensor node in the body segment can be determined.

Since there is no offset calculation on the axis, it implies that the module will always be considered to have the calculated axis with more weight facing the gravity, when considering its maximum value $(+1 \mathrm{~g})$. This can be problematic if the sensor node is not attached correctly to the chosen body segment; to tackle this issue, offset calculations will be introduced in the future.

\subsubsection{Body Segment Discovery}

The process of finding the location of the sensor nodes on the body starts immediately after the application concludes the orientation discovery process. A set of instructions (info messages) are given to the user, with the intent of guiding him through the procedure of determining the placement of the sensor nodes attached to the body. These guidance instructions consist of leading the user to make specific movements that allow the application to recognize the corresponding movements for each sensor node installed. Take for instance the case where only two sensor nodes are installed. When rehabilitating the arm, with the modules in the upper or lower member (forearm), the user is asked to move the forearm. Then the application detects which has the largest variation of movement. Even with a small movement, this is valid. The segment detection for the leg uses the same principle.

\section{ANGLE MEASUREMENT}

The accuracy of the sensor nodes, after on-site calibration, is limited by the sensor's noise, which adds to the system a measure of 1 degree error. Adding to this, it is known that accelerometer readings are influenced, not only by gravity, but also from acceleration during the motion of the body. 
This type of acceleration is not desirable, because it induces errors at the time of converting raw data from the sensors into angles. To tackle this problem, a compensation algorithm is applied to the accelerometer. Each accelerometer reading is composed by three values (one for each reading axis). If no linear acceleration is present (only the gravitational component), the vector magnitude must always equal to $1 \mathrm{~g}$. If this magnitude is not unitary under a certain tolerance, meaning that the measure contains linear acceleration besides gravity, a compensation algorithm is initiated. This algorithm uses the values from the current and previous magnetic readings to compensate for the linear acceleration errors. This method consists on using two consecutive magnetic readings (previous and current) to evaluate their angle $(\theta)$ and axis of rotation (axis), as shown in equations 1 and 2. The calculation of the angle and the axis of rotation, allows the rotation of the previous gravity vector to its new orientation (Dunn and Parberry, 2011).

$$
\begin{gathered}
\theta=\arccos \left(\vec{M}_{n-1} \cdot \vec{M}_{n}\right) \\
\text { axis }=\left(\vec{M}_{n-1} \times \vec{M}_{n}\right)
\end{gathered}
$$

The smallest angle between the two vectors (normalized previous and current magnetic readings) is provided by the dot product seen in equation 1 . This equation is valid since the magnitude of the magnetic vector is unitary. The cross product between those two vectors, equation 2, results on the rotation vector, around which the previous acceleration vector (gravity vector) is rotated.

The three angles that describe the orientation of the sensor nodes are obtained using the equations 3 , 4 and 5 respectively. The calculus of Pitch and Roll use the variations of the normalized accelerometer vector on each axis $\left(a_{x}, a_{y}, a_{z}\right)$. Yaw angle is obtained using only the horizontal components $\left(X_{h}, Y_{h}\right)$ of earth's magnetic field, previously calculated.

$$
\begin{gathered}
\text { Pitch }=\arctan \left(\frac{-a_{y}}{\sqrt{a_{x}^{2}+a_{z}^{2}}}\right) \\
\text { Roll }=\arctan \left(\frac{a_{x}}{-a_{z}}\right) \\
\text { Yaw }=\arctan \left(\frac{X_{h}}{Y_{h}}\right)
\end{gathered}
$$

The right-handed coordinate system was used to calculate the three orientation angles, as shown in Figure 7. The forward orientation of the module is set to be along the y axis, with the pitch defining the rotation on the $\mathrm{x}$ axis, the roll on the $\mathrm{y}$ axis and the yaw on the $\mathrm{z}$ axis.

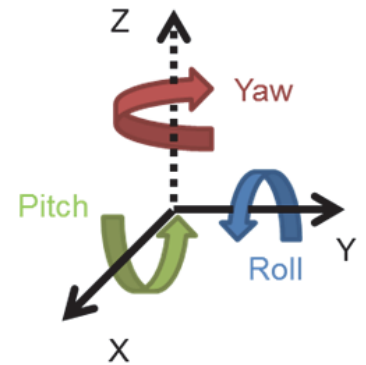

Figure 7: Right-handed coordinate system axis representation.

\section{EXPERIMENTAL RESULTS}

Experiments were carried out to test the developed system. One of them had two sensor nodes attached to the right arm (upper arm and forearm). The goal was to verify the replication of the body movement in the 3D model present in the application. Figure 8 and Figure 9 exemplify these experiments by means of photographs taken while the movement was being executed.

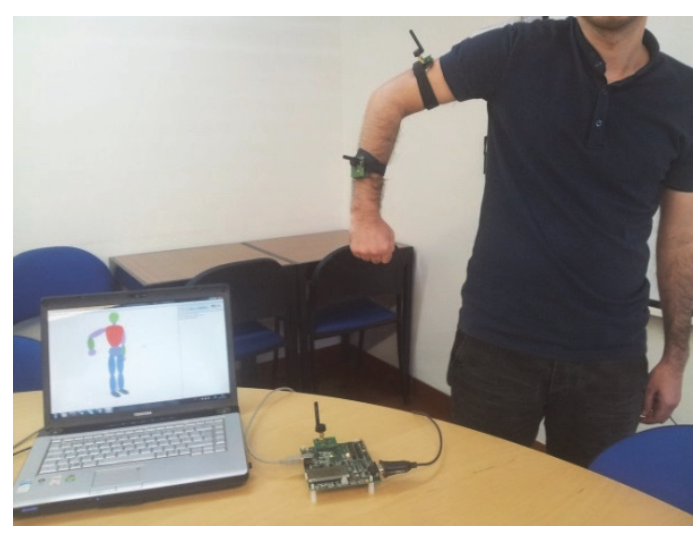

Figure 8: Right arm movement replication, first position.

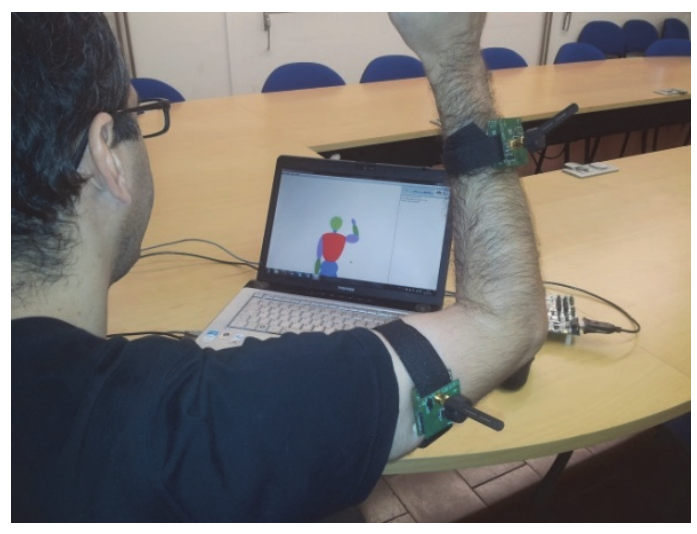

Figure 9: Right arm movement replication, second position. 
In order to simulate a rehabilitation session, a second test was performed. The purpose was to measure the angle between the upper and forearm. The user was requested to do a simple movement, flex and extend the arm, multiple times. The angle $(\varphi)$ between two joint connected body segments, in this case upper arm and forearm, is calculated using equation 6 .

$$
\varphi=\arccos \left(\frac{\vec{V}_{\text {upper }} \cdot \vec{V}_{\text {forearm }}}{\left\|V_{\text {upper }}\right\|\left\|V_{\text {forearm }}\right\|}\right)
$$

At this point, the application only deals with rehabilitation in both arms and legs, with two sensor nodes in the network. Figure 10 is a draft of what is desired in a session file. The wave variations seen on the right side reflect the angle variation (in degrees) between the body segments over time.

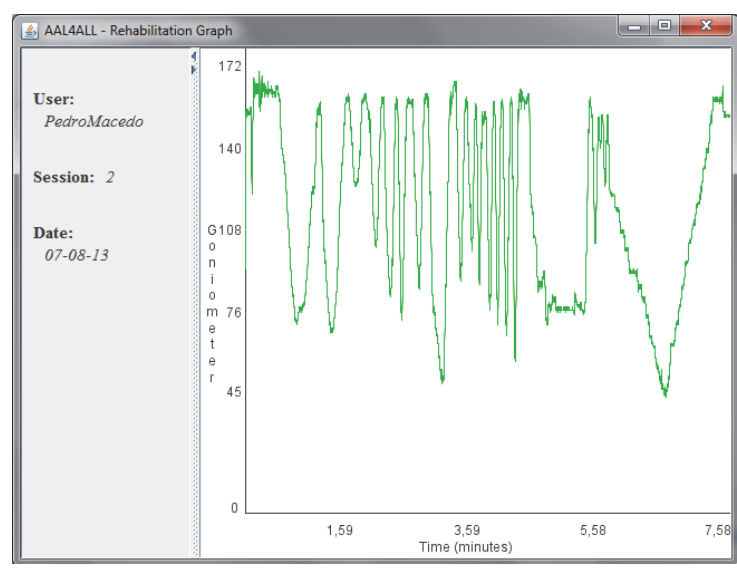

Figure 10: Rehabilitation application draft interface.

\section{CONCLUSIONS AND FUTURE WORK}

This paper describes early studies of the design and development of a wireless sensor network, based on wearable and easily placed inertial and magnetic sensors, when applied to physical activities. A Java application capable of collecting data from the sensors and present them in a real-time 3D model of a human body is under development, with the aim of assisting elderlies in the practice of physical activity.

At this stage, only the motion capture system capabilities can be evaluated. The displayed Java application does not transmit any kind of movement feedback; merely reproduces the executed motion. The main goal of this project consists in developing a home-based rehabilitation system that, through constant monitoring of the movement, is capable of interacting in real-time with the user. The objective of this interaction is to assist the user to perform the prescribed exercise correctly. Poorly executed exercises can delay the rehabilitation process, or even cause more damage. Thus, medical specialists (physiotherapists) should take into consideration physical limitation of the clinical subject in the preparation of physiotherapy sessions, and set welldefined objectives with regard to patient outcomes.

As future work it is intended that the patient, while doing the prescribed exercises at home, receives feedback from the application about the correctness of those movements. Also, future work includes inserting more sensor nodes in the network, in order to obtain (simultaneously) the angle between several joint connected segments, thus allowing better assessing the user's performance. The application graphical interface will be improved, simplifying it and giving more emphasis to the 3D model of the human body and rehabilitation graph.

Studies are being carried out in order to facilitate the automatic body segment recognition for more than two sensor nodes. As the number of nodes increases, the number of required movements also increases, and recognition will have to occur in separate steps. In certain physical activities, it is advisable to have one sensor node attached to the torso, as it can provide a more accurate perspective of the entire motion sequence.

Studies are also being conducted to introduce the 3 -axis MEMS gyroscope present in the MPU-6000 in the module's orientation calculation. The use of the gyroscope allows compensation (not elimination) of the linear acceleration detected by the accelerometer and compensates false angular movements.

The natural evolution of this system includes also the development of a single, smaller board containing both the CC2530 and the sensors, as well as a printed circuit antenna to replace the existing external antenna.

\section{ACKNOWLEDGEMENTS}

Project "AAL4ALL", co-financed by the European Community Fund FEDER through COMPETE Programa Operacional Factores de Competitividade (POFC). FCT - Foundation for Science and Technology - Lisbon, Portugal, through project PEst-C/CTM/LA0025/2013. 


\section{REFERENCES}

Afonso, J., Silva, H., Macedo, P., Rocha, L., 2011. An Enhanced Reservation-Based MAC Protocol for IEEE 802.15.4 Networks. Sensors, Vol. 11, Issue 4, April 2011, pp. 3852-3873.

Aminian, K., Najafi, B., 2004. Capturing human motion using body-fixed sensors: outdoor measurement and clinical applications. In Computer Animation and Virtual Worlds, Volume 15, Issue 2, pp. 79-94.

Cutti, A., Giovanardi, A., Rocchi, L., Davalli, A., Sacchetti, R., 2007. Ambulatory measurements of shoulder and elbow kinematics through inertial and magnetic sensors. In Medical \& Biological Engineering \& Computing, Volume 46, Issue 2, pp. 169-178.

Claypool, M., Claypool, K., Damaa, F., 2006. The Effects of FrameRate and Resolution on Users Playing First Person Shooter Games. In Proceedings of a ACM/SPIE Multimedia Computing and Networking $(M M C N)$.

Dunn, F., Parberry, I., 2011. 3D Math Primer for Graphics and Game Development. Second Edition pp.141-143, ISBN-13: 978-1-4398-6981-9.

Farella, E., Pieracci, A., Benini, L., Rocchi, L., Acquaviva, A., 2008. Interfacing human and computer with wireless body area sensor networks: the WiMoCA solution. In Multimedia Tools and Applications, Volume 38, Issue 3, pp. 337-363.

Fuchsberger, M., 2008. Ambient Assisted Living: Elderly People's Needs and How to face Them. In Proceedings of the $1^{\text {st }}$ ACM international workshop on Semantic ambient media experiences.

Hadjidj, A., Souil, M., Bouadballah, A., Challal, Y., Owen, H., 2012. Wireless sensor networks for rehabilitation applications: Challenges and opportunities. In Journal of Network and Computer Applications.

IEEE Std 802.15.4-2006, Part 15.4: Wireless Medium Access Control (MAC) and Physical Layer (PHY) Specifications for Low-Rate Wireless Personal Area Networks (WPANs), September 2006.

Kleinberger, T., Becker, M., Ras, E., Holzinger, A., 2007. Ambient Intelligence in Assisted Living: Enable Elderly People to Handle Future Interfaces. In $4^{\text {th }}$ International Conference on Universal Access in Huma-Computer Interaction, UAHCI.

Linz, K., Stula, S., 2012. Demographic change in Europe An Overview. Working Paper No. 4 of the Observatory for Sociopolitical Developments in Europe.

Moeslund, T. B., Granum, E., 2001. A survey of computer vision-based human motion capture. In Computer Vision and Image Understanding.

Steffen, D., Bleser, G., Weber, M., Stricker, D., Fradet, L., Marin, F., 2011. A personalized Exercise Trainer for Elderly. In $5^{\text {th }}$ International Conference on Pervasive Computing Technologies for Healthcare (PervasiveHealth) and Workshops.
Stula, S., 2012. Living in Old Age in Europe - Current Developments and Challenges. Working Paper No. 7 of the Observatory for Sociopolitical Developments in Europe.

Sun, H., De Florio, V., Gui, N., Blondia, C., 2009. Promises and Challenges of Ambient Assisted Living Systems. In Information Technology: New Generations. ITNG'09.

Texas Instruments, "CC2530 data Sheet" April 2009. Retrieved from http://www.ti.com

Wang, L., Hu, W., Tan, T., 2003. Recent developments in human motion analysis. In Pattern Recognition, Volume 36, Issue 3, pp. 585-601.

Wong, W., Wong, M., Lo, K., 2007. Clinical Applications of Sensors for Human Posture and Movement Analysis: A Review. In International Society for Prosthetics and Orthotics. 